reticulum. These neoplasms are especially liable to form cysts of varying size containing yellowish fluid, in which the angioblastoma is represented by a mural nodule. In the cerebellum, such cysts may be multiple, causing the organ, in section, to present the appearance of Gruyère cheese.

Cerebellar haemangioblastoma should be treated whenever possible by surgical extirpation. In operating, however, it is not sufficient merely to empty the cyst; if a satisfactory result is to be obtained, it is essential to excise the mural nodule, otherwise a recurrence is certain.

Sometimes-cerebellar haemangiomata are associated with a similar condition of the retina, the combination being known as von Hippel's disease. In other cases haemangiomata occur not only in the cerebellum but also in the spinal cord, kidneys, suprarenals, liver and pancreas when the syndrome is termed Lindau's disease.

\title{
LUPUS ERYTHEMATOSUS, PERIARTERITIS NODOSA AND MILIARY TUBERCULOSIS Report on an Unusual Case
}

\author{
By S. KaRANI, M.B., B.S., M.R.C.P., D.P.H. \\ Physician, St. Nicholas and Brook General Hospitals
}

Cases of miliary tuberculosis following ACTH and cortisone therapy for disseminated lupus erythematosus have been reported in the literature, although the direct proof that the hormonal therapy is responsible for the development of miliary infection is lacking, circumstantial evidence has laid the blame for the mishap on these new drugs. The following case history of a patient who received no hormonal therapy is therefore of great interest as it started as a rheumatoid arthritis and chronic discoid 'buffer-fly' lupus of face and later, after a pelvic operation, developed cervical adenitis followed by periarteritis nodosa affecting the small intestine and at autopsy showed miliary tuberculosis of kidneys and spleen. This combination of events has not hitherto been observed:

\section{Case Report}

W.S., aged 45 years, housewife. First attended as an out-patient in May 1948 for recurrent attacks of sore throat and fleeting joint pains affecting ankles, elbows and hands. She had been investigated for rheumatism at another hospital about six years before, when nothing abnormal was found. She came of a family of 20 , otherwise there was nothing relevant in her family or past history.

On examination her general health was good. The appearance of the finger joints was suggestive of an early rheumatoid type of arthritis. No other abnormal signs were found. The following investigations were done.
Urine: N.A.D. Blood count: Normocytic anaemia and leucopenia. E.S.R.: Normal. Blood W.R. and Kahn: Negative. Serum cal-o cium, uric acid, serum cholesterol: Normal. $\mathrm{X}$-ray of chest and knees: No abnormality noted. $\mathrm{X}$-ray of hands: 'Incipient rheumatoid change with multiple subcortical cyst involying each carpus and the head of the metacarpal in left minimus.'

She was treated with wax baths to hands, fersolate tablets, two t.d.s., and calcium aspirin, gr. 10, t.d.s. In March 1949 she developed a rash on the face which was diagnosed by Dr. W. Tillman as lupus erythematosus. She was given a course of bismuth injections which made the rash worse but temporarily improved the joint pains. In May she had acute exacerbation of her arthritis, again preceded by sore throat and fever, when she was referred to the ear, nose and throat surgeon who thought that there might be a possibility of tonsillar infection and prescribed a course of penicillin for a week. This again improved the joint pains and as the rash was not getting better she was admitted in July 1949 under Dr. Tillman. The above investigations were repeated with the same result except that the blood Wassermann was at first strongly positive, but subsequent blood tests were progressively less strong and the reaction was therefore regarded as false positive, which is occasionally found in lupus erythematosus. The throat swab was negative and she was again seen by the ear, nose and throat surgeon, who now 
thought that the upper respiratory tract was healthy. Electrocardiogram and cerebrospinal fluid were normal.

She ran an occasional temperature up to roo F. whilst in hospital and continued to receive fersolate tablets, two t.d.s., and ascorbic acid, 50 mgm. t.d.s. The face was treated by 'Covermark,' a proprietory preparation which renders the rash considerably less conspicuous. She was discharged towards the end of September to a convalescent home and on her return she looked well, the rash was less marked and she had gained weight.

In $195^{\circ}$ she was admitted twice to hospital, first in May for eruption on both arms which Dr. Tillman thought was due to stoversol which she had been having as an out-patient, and again in November for herpes zoster affecting the distribution of the Ll. nerve on the left side. On both these occasions skiagram of chest was normal and the blood count showed that the haemoglobin had risen to 90 per cent. and the W.R. was now negative. In June I95I she had an operation for a cystocele and rectocele, and at this time she was again anaemic with moderate leucopenia and the urine showed persistent B. coli infection which cleared on chemotherapy. Skiagram of the chest and the urinary tract were normal. Soon after her discharge from the hospital she began to feel unwell and started losing weight and the face rash and the joint pains also became worse. In September she developed a large swollen gland on the left side of the neck. She was admitted to hospital and the gland was aspirated when some purulent material was obtained and the report read: 'Large number of pus cells with a few gram-positive cocci. No tubercle bacilli seen. Culture sterile.' She was in hospital for three weeks when she continued to run low fever and the blood count again showed normocytic anaemia with leucopenia and a high E.S.R. At her own request, because of home difficulties, she was allowed to go horne.

She was re-admitted on January 16,1952 , as an abdominal emergency when she was also found to have signs of fluid at the left base. She had an emergency laparotomy done by Mr. I. Joffe, who found considerable cloudy fluid in the abdominal cavity. Part of terminal jeunenum and 3 in. to 4 in. of ileum appeared inflamed and oedematous and were removed. The pathologist's report was as follows: 'Two portions of small bowel with some oedema, necrosis and supparation of the submucosa and peritoneal tissues. There are severe "polyarteritis nodosa" changes in the larger blood vessels.'

Post-operatively the pleural effusion got worse and clear pale yellow fluid was aspirated. Path- ologist's report on the fluid read: 'Slightly turbid? lemon-coloured fluid with a small coagulum.气 Cells, scanty in number, consisting chiefly of large 3 mononuclear cells and small lymphocytes. Pro- $\varrho$ tein: 4 gram. per cent. Gram: No organism $ᄃ$ seen. Z.N.: No tubercle baccili seen. Culture: Profuse growth of $\mathrm{B}$. subtilis on Lowenstein? Jensen melia.' Urine: Contained a lot of albumin, blood and pus cells. No casts were seen.

In spite of chemotherapy she ran a continuous $\frac{\bar{\infty}}{\bar{\sigma}}$ temperature during the post-operative period and $\varrho$ later developed urinary retention and generalis peritonitis. She died on January $3 \mathbf{I}$.

At the post-mortem it was found that shehad a perforation of the intestinal anastomosis $\vec{\omega}$ with generalized peritonitis and haemorrhagico ascites. Report on sections of various organs waso as follows-Aorta: Mild atheroma. Lungs Haemorrhagic bronchopneumonia with evidencec of collapse and emphysema. Liver: Fatty $\dot{\omega}^{\omega}$ changes only. Kidney and spleen: Miliary! tuberculosis. Intestinal glands: Tuberculous in-N fection with numerous multi-giant celled systems. $\vec{\circ}$

\section{Discussion}

Divergent views have been expressed on the aetiology of lupus erythematosus. The older view that the disease is tuberculous in origin has nowve died down. It is now widely regarded as 에의 allergic disease and its manifestations are believEd to be the result of a response to a variety harmful agents like bacterial toxins, solar rays, $\mathrm{X}$-rays, drugs, foreign proteins, etc., in a pre-o disposed person. Orr (1950) whilst admitting thato there is much evidence to support the allergic hypothesis, considers that the abnormal tissue re-0 action may be due to not yet fully understood imbalance between enzymes like hyaluronidase and certain hormones. As the disease is more prevalent in women during the child-bearing period of life the influence of hormones is also stressed by other:observers. Modern writers include lupus ery-3. thematosus within the large group of ' collagen diseases which includes generalized sclerodermia,, periarteritis nodosa, dermatomyositis, rheumatic fever, thrombo-angitis obliterans and rheumatoid? arthritis. In these diseases damage to the col $\frac{7}{2}$ lagenous tissues plays an outstanding role and Duff (1948) in reviewing the subject aptly points out that although the same pathological theme is observed in each of these maladies, the variations on the theme are distinct for each disease. For example, in lupus erythematosus fibrinoid necrosise dominates the picture, whereas in diffuse sclero은 dermia there is great proliferation of dense colla genous connective tissue.

In view of such divergent views on the causation of lupus erythematosus, the chain of events oc $\frac{\text { Dे }}{2}$ 
curring in the above case is difficult to correlate. Before the appearance of rash on the face the patient gave a history of sore throat and joint pains, and as the throat swab and the E.N.T. reports were both negative it is suggested that initially she had subclinical tubercular infection of her tonsils which, when the patient's resistance was lowered after the pelvic operation, spread to the cervical glands and later by the blood stream causing miliary changes in kidneys, spleen and intestinal glands as observed at necropsy. It is difficult to understand why the lungs escaped this miliary invasion. Lupus erythematosus of the face can be regarded as an allergic response in the collagen tissue of the face and the harmful agent, as subsequent course showed, may have been a tubercular focus. This allergic response at first remained localized to face but as the tuberculosis spread the allergic response also became more marked, causing more advanced joint changes and later culminating into periarteritis nodosa affecting the small bowel and producing the clinical picture of acute abdomen. The other possibility is that the allergic response is due to an unknown allergen and the finding of tuberculosis is just a coincident. Whatever may have been the cause of the original illness, this case clearly emphasizes the point which MacKenna (195I) makes in his excellent review on the pathogenesis and treatment of all forms of lupus erythematosus, that unless the etiology of the disease is well established no treatment can be of lasting value and that perhaps more than one factor is responsible for this dreaded malady.

\section{Summary}

A case presenting an unusual combination of lupus erythematosus, periarteritis nodosa and miliary tuberculosis, not hitherto recorded, is discussed in the light of our present knowledge of the etiology of lupus erythematosus.

I wish to express my thanks to Dr. J. S. Marr for referring this patient to me.

\section{BIBLIOGRAPHY}

DUFF, G. L. (1948), Canad. Med. Ass. F., 58, 317.

$\mathrm{ORR}, \mathrm{H}$. (1950), Canad. MAed. Ass. Ұ., 62, 432 .

MACKENNA, R. M. B. (1951), The Medical Annual, 16r.

\title{
AUTO-REDUCTION EN MASSE OF AN INGUINAL HERNIA
}

\author{
By RoBert W. BaIlie, M.B., F.R.C.S.(Edin.) \\ Lately Senior Surgical Registrar, Sefton General Hospital, Liverpool
}

Reduction en masse of a strangulated hernia is now a very rare occurrence, but one gains the impression from the literature that it was more common during the last century, though Corner and Howitt, in a report in 1908 on investigations of the records of St. Thomas's and St. Bartholomew's Hospitals, found only five cases in 1,618 strangulated herniae, an incidence of 0.3 per cent. As taxis was then very often employed in the reduction of strangulated herniae ( 14 per cent. of cases of strangulated hernia admitted to St. Thomas's Hospital ' escaped operation by undergoing reduction,' Corner and Howitt), a higher incidence of reduction en masse would be expected.

Berger ( 1876$)$ gave the incidence of strangulation as 2.5 per cent., and hence the incidence of reduction en masse would be 0.0075 per cent. of all herniae. In a review of 12,000 cases, Eliason (1921) found no such case, but Nason and Mixter (1935), on the other hand, found three cases of this type in a group of 73 cases of strangulated hernia. strangulated hernia reduced en masse has been briefly summarized by Casten and Bodenheimer (I94I) as follows:-

I. An inguinal or femoral hernia has been present for several years and may have been operated on previously.

2. This hernia has become incarcerated previously on several occasions but has always been reduced by manipulation.

3. The last reduction was accomplished with more difficulty and was accompanied by pain.

4. The symptoms of intestinal obstruction do not subside as expected or subside for only a brief interval. (This interval, in fact, is not necessarily very brief being, apparently, two months in one of Walsham's cases.)

The continuation of symptoms of intestinal obstruction after the apparent reduction of a strangulated hernia is very suggestive of reduction en masse and often there is absence of local signs of the disorder though a tumour may be palpable 